\title{
Value of Arterialized Capillary Blood Gas Analysis in Lower Respiratory Tract Infection in Childhood
}

\author{
W. B. DOIG \\ From the Department of Child Health, University of Glasgow, Royal Hospital for Sick Children, Glasgow
}

\begin{abstract}
Doig, W. B. (1971). Archives of Disease in Childhood, 46, 243. Value of arterialized capillary blood gas analysis in lower respiratory tract infection in childhood. Blood gas analysis on arterialized capillary blood in 70 young infants yielded satisfactory information about oxygen therapy. Inspired oxygen concentrations above $40 \%$ are often required to raise the capillary $\mathrm{Po}_{2}$ of hypoxic infants above $70 \mathrm{mmHg}$. Oxygen concentrations above $40 \%$ are difficult to achieve and maintain in presently available oxygen tents, though not in modern incubators, or oxygen chairs. A raised $\mathrm{PCO}_{2}$ has frequently been found in infants with severe respiratory infections, but oxygen therapy has not resulted in $\mathrm{CO}_{2}$ narcosis in these acute emergencies.
\end{abstract}

Acute infections of the lower respiratory tract (LRTI) such as acute bronchiolitis and bronchopneumonia constitute a common problem in infancy. They vary widely in degree of severity, but nursing in a humid and oxygen enriched atmosphere is often indicated. Since the degree of hypoxia endured by these infants may be greater than that suggested by the clinical assessment of cyanosis and respiratory difficulty, it is desirable to be able to monitor oxygen therapy by measuring blood gases, relating $\mathrm{Po}_{2}$ level to the ambient oxygen concentration. Only by so doing can it be certain that oxygen is required and is being given in adequate concentration. Unfortunately the technical problems have all too often resulted in the dyspnoeic infant whose oxygen tension is unknown being nursed in a tent where the oxygen concentration is also unknown. In the neonatal period the accessibility of the umbilical arteries means that arterial $\mathrm{Po}_{2}$ levels can be measured with relative ease; in older children and adults arterial blood may be obtained by arterial puncture or by the insertion of an indwelling catheter. In infancy, however, arterial sampling is more difficult. Arterialized capillary blood obtained after suitable preparation of the capillary bed is an alternative to arterial puncture, and we here describe our experience with this technique in $\mathbf{7 0}$ infants.

Received 23 October 1970.

\section{Material and Methods}

Seventy infants with ages ranging from 17 to 133 days admitted to hospital with acute LRTI of varying severity were studied. Samples for blood gas analysis were obtained on admission in 66 instances while the infants were breathing air; in 4 cases oxygen was already being given at the time of sampling. In 26 cases both arterial and capillary samples were obtained at the initial examination while the infants were breathing air. Capillary samples were taken from the heel; the chosen site was prepared by rubbing for 4 minutes with thurfyl nicotinate (Trafuril). While waiting for the heel to flush arterial sampling was attempted. The radial artery was preferred and samples were withdrawn into heparinized syringes by direct stab. Capillary samples were then obtained by incising the prepared heel with a Sterolet; blood was collected into $100 \mu \mathrm{l}$ heparinized glass capillary tubes (Radiometer type D.551) and the ends promptly sealed with plasticine. Thurfyl nicotinate preparation in this way was found to yield a very satisfactory free flow of blood. All measurements were made within 15 minutes of sampling. $\mathrm{PO}_{2}$ measurements were made with the Radiometer oxygen microelectrode; $p \mathrm{H}, \mathrm{PCO}_{2}$, standard bicarbonate, and base excess were measured by the Astrup method.

Capillary $\mathrm{PO}_{2}$ was determined in a control group of 50 babies not suffering from a condition likely to cause hypoxia: the opportunity to obtain samples was taken when blood was required for other investigations (e.g. Hb, WBC, serum calcium etc.). Thurfyl nicotinate preparation was used.

According to the initial results and the degree of hypoxia, patients were nursed in air alone, air with 
humidity or in humidity with oxygen enrichment using incubators (Vickers Medical) or Isolette (Air-Shields), small croupettes (Air-Shields), Humidaire tents (Vickers Medical), or oxygen chairs (Gomez et al., 1968). Oxygen enrichment was monitored hourly using a paramagnetic oxygen analyser (Mira). Repeat analyses were obtained from capillary samples as described. When infants were nursed in tents the heel could be withdrawn beneath the canopy without disturbing the infant's atmosphere; if an incubator were used, a porthole was sealed with a sheet of polythene 30 minutes before sampling, at which time a small hole was made in the sheeting through which the heel was withdrawn: in this way the atmosphere in the incubator was maintained. If an oxygen chair was used, samples were obtained with no disturbance to the infant's environment. Nursing care of the infants requires opening of all apparatus from time to time, but handling was kept to a minimum to avoid loss of oxygen concentration. No sample in this series was taken unless the infant had been in his environment undisturbed for at least 30 minutes.

When an infant is stabbed he cries and may breathhold long enough to cause an abrupt fall in $\mathrm{Po}_{2}$ level. Indeed in obtaining samples, whether arterial or capillary, a colour change in the blood was often visible during the course of the sampling. Some infants who were not cyanosed and only mildly dyspnoeic when undisturbed became obviously cyanosed on sampling, so that the resultant $\mathrm{PO}_{2}$ was clearly not truly representative.

Where oxygen therapy was indicated, $35-40 \%$ was in most cases adequate. In severe hypoxia over $50 \%$ oxygen had to be given for satisfactory relief. Such high levels are obtained quite readily in oxygen chairs (Gomez et al., 1968) and incubators, but even with high oxygen flow rates were difficult to maintain in tents despite manufacturer's claims.

\section{Results}

The $\mathrm{Po}_{2}$ range in the 50 controls was $62-99$ mmHg (Fig. 1); 38 infants had $\mathrm{Po}_{2}$ 's of $70 \mathrm{mmHg}$ or greater. A level of less than $70 \mathrm{mmHg}$ in an infant with LRTI was taken as indicating the probable need for oxygen therapy.

$\mathrm{Po}_{2}$ levels in the 26 patients with LRTI in whom both arterial and capillary samples were obtained are compared in the Table. In 15 instances the arterial level was greater than the capillary, the greatest difference being $18 \mathrm{mmHg}$, but in 10 instances the capillary level exceeded the arterial, the greatest difference being $13 \mathrm{mmHg}$; in only one instance were the two identical. These samples were obtained at the same session though not simultaneously. No attempt is made here to compare arterial and capillary $\mathbf{P o}_{2}$ levels statistically, but the differences probably reflect the difficulty in obtaining a resting state in this age group.

In 66 of the 70 patients the initial capillary

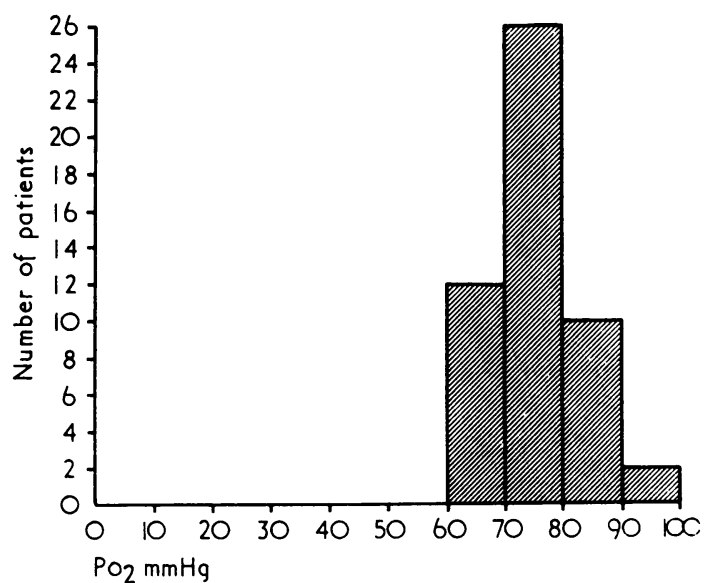

Fig. 1.- Histogram showing the range of $\mathrm{PO}_{2}$ in arterialized capillary blood samples from 50 normal infants.

TABLE

Initial Arterial and Capillary $\mathrm{Po}_{2}$ levels in 26 Infants with Lower Respiratory Tract Infection

\begin{tabular}{|c|c|c|}
\hline $\begin{array}{l}\text { Arterial } \mathrm{Po}_{2} \\
(\mathrm{mmHg})\end{array}$ & $\begin{array}{l}\text { Capillary } \mathrm{Po}_{2} \\
\quad(\mathrm{mmHg})\end{array}$ & $\begin{array}{l}\text { Arterial-Capillary } \\
\text { Difference }\end{array}$ \\
\hline $\begin{array}{l}82 \\
66 \\
62 \\
89 \\
44 \\
71 \\
84 \\
76 \\
52 \\
63 \\
75 \\
68 \\
57 \\
80 \\
60 \\
71 \\
78 \\
77 \\
70 \\
72 \\
60 \\
78 \\
59 \\
72 \\
69 \\
64\end{array}$ & $\begin{array}{l}64 \\
52 \\
57 \\
77 \\
53 \\
62 \\
67 \\
67 \\
46 \\
55 \\
72 \\
64 \\
58 \\
87 \\
73 \\
65 \\
81 \\
72 \\
74 \\
68 \\
72 \\
79 \\
59 \\
75 \\
74 \\
63\end{array}$ & $\begin{array}{r}+18 \\
+14 \\
+5 \\
+12 \\
-9 \\
+9 \\
+17 \\
+9 \\
+6 \\
+8 \\
+3 \\
+4 \\
-1 \\
-7 \\
-13 \\
+6 \\
-3 \\
+5 \\
-4 \\
+4 \\
-12 \\
-1 \\
0 \\
-3 \\
-5 \\
+1\end{array}$ \\
\hline
\end{tabular}

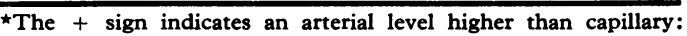
the - sign the reverse.

$\mathrm{Po}_{2}$ level was obtained with the infant breathing air (Fig. 2). The range was $22-87 \mathrm{mmHg}$ and in 42 cases was less than $70 \mathrm{mmHg}$. 36 of these 42 cases were at once nursed in oxygen. The initial oxygen concentration for the $\mathbf{4 0}$ infants so treated ranged from $30-60 \%$ and the corresponding $\mathrm{Po}_{2}$ 


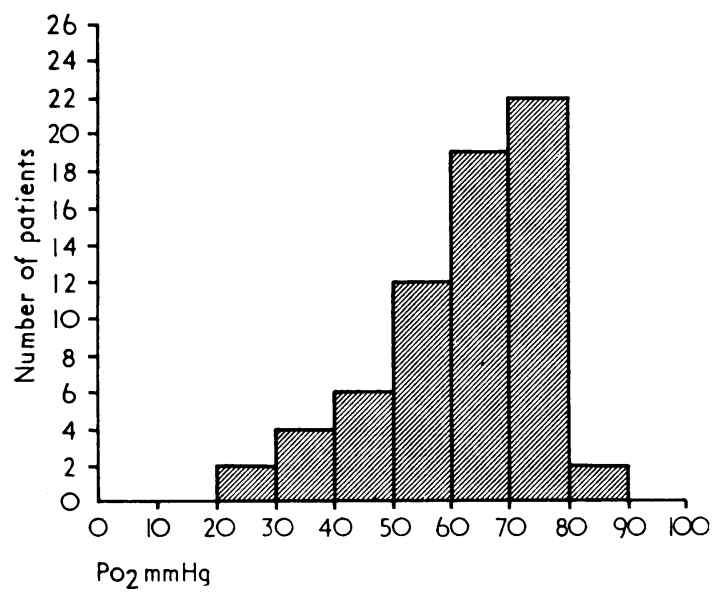

FIG. 2.-Histogram showing the range of $\mathrm{PO}_{2}$ in arterialized capillary blood samples in 66 cases of lower respiratory tract infection (breathing air).

levels were $47-118 \mathrm{mmHg}$. 27 infants were in an adequate concentration $\left(\mathrm{Po}_{2} 70 \mathrm{mmHg}\right.$ or more) but 13 still had a $\mathrm{Po}_{2}$ of less than $70 \mathrm{mmHg}$. In 2 cases an increase of oxygen to a level still less than $60 \%$ was adequate, but in 11 cases a concentration greater than $60 \%$ was required to maintain a $\mathrm{Po}_{2}$ of greater than $70 \mathrm{mmHg}$ and 2 infants required oxygen for 12 days or more.

Most infants had a degree of hypercarbia: in air the initial $\mathrm{PCO}_{2}$ levels ranged from $30-100 \mathrm{mmHg}$, and 38 infants had levels of $45 \mathrm{mmHg}$ or more. During oxygen therapy the highest rise in $\mathrm{PCO}_{2}$, over the initial value obtained after admission, was $15 \mathrm{mmHg}$ and the highest recorded level was 105
mmHg. By discharge the $\mathrm{PCO}_{2}$ in all cases was normal $(<38 \mathrm{mmHg})$. Assisted ventilation was not employed in any infant in this series. There were no deaths.

\section{Illustrative Case Reports}

Case 1 (Fig. 3). On admission this 2-month-old infant had signs of severe acute bronchiolitis and was in oxygen when first examined. In an initial oxygen concentration of $30 \%$ her $\mathrm{Po}_{2}$ was obviously not satisfactory being only $47 \mathrm{mmHg}$, and increasing the concentration to $46 \%$ had little effect $\left(\mathrm{Po}_{2}-45 \mathrm{mmHg}\right)$. To obtain a satisfactory $\mathrm{Po}_{2}$ she had to be nursed in a concentration of oxygen of $60 \%$ or higher for 10 days and at a somewhat lower concentration for a further 2 days before she maintained a $\mathrm{Po}_{2}$ above $70 \mathrm{mmHg}$ in air alone. 17 samples were obtained from her, 7 during the first 48 hours during which time the required environmental oxygen concentration progressively increased.

Case 2 (Fig 4). Admitted aged $2 \frac{1}{2}$ months with moderately severe acute bronchiolitis. On admission he was put straight into oxygen-enriched air and his initial $\mathrm{Po}_{2}$ of $118 \mathrm{mmHg}$ indicated that the environmental concentration of $40 \%$ was adequate. After 48 hours he was nursed in air, but as his $\mathrm{Po}_{2}$ fell to $56 \mathrm{mmHg}$ and his clinical condition deteriorated oxygen was restarted. On day 5 he was again nursed in air and was clinically much improved: during sampling however he breathheld and his $\mathrm{Po}_{2}$ of $55 \mathrm{mmHg}$ was clearly not truly representative of his resting state. No further oxygen was given and he continued to improve steadily.

\section{Discussion}

Infants with LRTI are often more hypoxic than simple clinical assessment would suggest. In

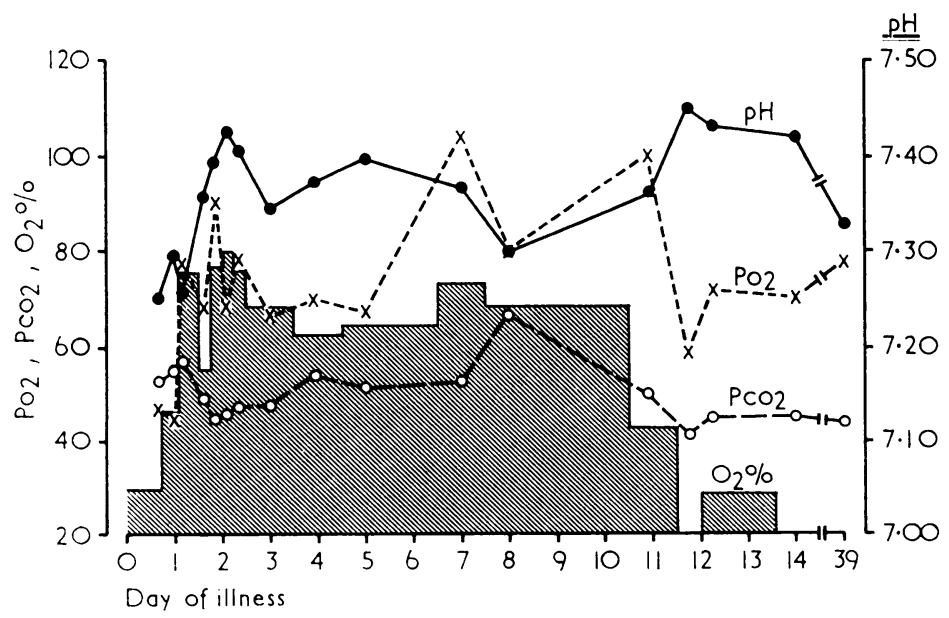

Fig. 3.-Blood gas findings during oxygen therapy in a 2-month-old infant with severe acute bronchiolitis. 


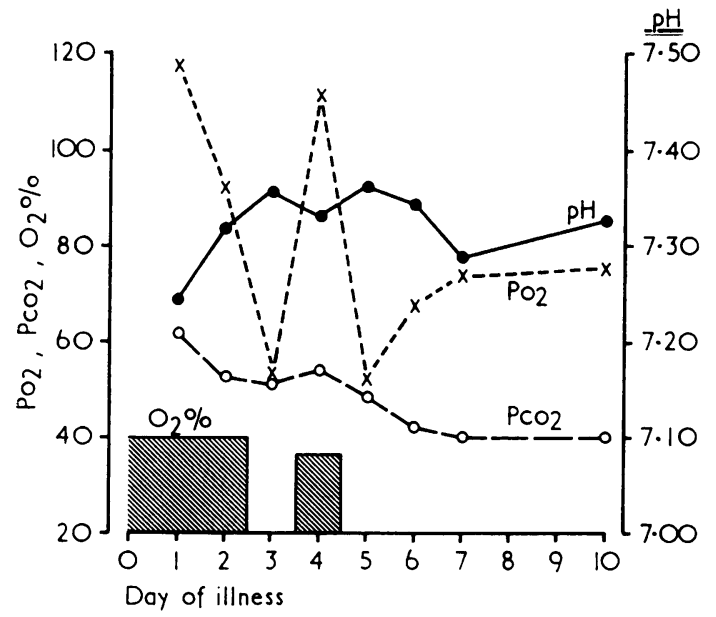

Fig. 4.-Blood gas findings during oxygen therapy in a 21-month-old infant with acute bronchiolitis.

deciding on the need for, and in monitoring the effect of oxygen therapy, arterial blood sampling is ideally required. While methods of arterial puncture in infants have been described (Shaw, 1968; Bucci et al., 1966), there are undoubted difficulties in this age group and repeated sampling may be particularly difficult. In adults $\mathrm{Po}_{2}$ values for capillary blood agree closely with those obtained from arterial blood, provided that the site of sampling is adequately prepared (MacIntyre, Norman, and Smith, 1968). In infants the use of thurfyl nicotinate produces a very satisfactory free flow of capillary blood and the heel may be used for repeated sampling during therapy. A major problem in infants lies in their ability to co-operate, so that sampling leads often to breathholding and temporary increase in hypoxia. In this situation $\mathrm{Po}_{2}$ analysis should be repeated after a short interval if doubt exists as to the true significance of the initial value. Our aim has been to obtain a capillary $\mathrm{Po}_{2}$ level of $70 \mathrm{mmHg}$ or more. If this was obtained on admission, oxygen therapy was considered unnecessary in the first instance. In some cases subsequent deterioration indicated the need for resampling, and oxygen therapy did sometimes then become necessary.
If the capillary $\mathrm{Po}_{2}$ on admission was less than $70 \mathrm{mmHg}$ in the absence of breathholding, oxygen was then given. In many of these infants an oxygen concentration of $35-40 \%$ was adequate but as has been previously clearly shown (Simpson and Flenley, 1967) some infants require substantially higher concentrations to relieve hypoxia. It is noteworthy also that with the exception of incubators and oxygen chairs which are only suitable for the smallest infants, no tent presently available can reliably and consistently give an atmosphere of much more than $50 \%$ oxygen, an observation made by Simpson and Russell in 1967. There remains a need for an improved tent for large infants who require high oxygen concentration in association with high humidity.

Measurement of $\mathrm{Po}_{2}$ levels in arterialized capillary blood, though less reliable than arterial, allows satisfactory control of oxygen therapy in the young infant when the oxygen concentration in the inspired atmosphere is also monitored. Blood can always be obtained and can be frequently sampled. Oxygen therapy can obviously be more accurately controlled when such measurements are available, and hypoxia more confidently corrected.

My thanks are due to Professor J. H. Hutchison for his help, to the nursing staff of the Professorial Unit, Royal Hospital for Sick Children, Glasgow, and to Mr. J. Devlin and staff of the Department of Medical Illustration, Royal Hospital for Sick Children, Glasgow.

REFERENCES

Bucci, G., Scalamandrè, A., Savignoni, P. G., Orzalesi, M., and Mendicini, M. (1966). Crib-side sampling of blood from the radial artery. Pediatrics, 37, 497.

Gomez, P. C. W., Keaney, J. P. D., Rogers, K. G., and Carter, D. G. (1968). An oxygen chair. Archives of Disease in Childhood, 43, 738.

MacIntyre, J., Norman, J. N., and Smith, G. (1968). Use of capillary blood in measurement of arterial $\mathbf{P O}_{2}$. British Medical fournal, 3, 640 .

Shaw, J. C. L. (1968). Arterial sampling from the radial artery in premature and full-term infants. Lancet, 2, 389.

Simpson, H., and Flenley, D. C. (1967). Arterial blood-gas tensions in $\mathrm{pH}$ in acute lower-respiratory-tract infections in infancy and childhood. Lancet, $1,7$.

Simpson, H., and Russell, D. J. (1967). Oxygen concentrations in tents and incubators in paediatric practice. British Medical fournal, 4, 201.

Correspondence to Dr. W. B. Doig, Royal Hospital for Sick Children, Oakbank, Glasgow C.4. 